\title{
Real-time broad-range PCR versus blood culture. A prospective pilot study in pediatric cancer patients with fever and neutropenia
}

\author{
Roland A. Ammann • Franziska Zucol • \\ Christoph Aebi • Felix K. Niggli • Thomas Kühne • \\ David Nadal
}

Received: 3 November 2006 / Accepted: 8 February 2007 / Published online: 1 March 2007

(C) Springer-Verlag 2007

\begin{abstract}
Materials and methods In a pilot study, results of real-time broad-range (16S rRNA) polymerase chain reaction (PCR) performed on 45 blood samples of pediatric cancer patients with fever and neutropenia were compared with blood culture results.

Results The PCR assay used, having proven a high sensitivity in artificially spiked blood samples, was positive
\end{abstract}

Provisional results of this study were presented in part at the 36th conference of the International Society of Pediatric Oncology (SIOP) in Oslo, September 2004 (Abstract PD.017).

\author{
R. A. Ammann $(\bowtie)$ \\ Pediatric Hematology/Oncology, \\ Department of Pediatrics, University of Bern, \\ CH-3010 Bern, Switzerland \\ e-mail: roland.ammann@insel.ch \\ F. Zucol $\cdot$ D. Nadal \\ Division of Infectious Diseases, \\ University Children's Hospital, University of Zurich, \\ Zurich, Switzerland \\ C. Aebi \\ Institute of Infectious Diseases, University of Bern, \\ Bern, Switzerland \\ F. K. Niggli \\ Division of Oncology, University Children's Hospital, \\ University of Zurich, \\ Zurich, Switzerland \\ T. Kühne \\ Pediatric Hematology/Oncology, University Children's Hospital, \\ University of Basel, \\ Basel, Switzerland
}

in only three of ten blood culture-positive samples, and it was positive in 10 of $35(29 \%)$ culture-negative samples. Conclusion This broad-range PCR assay, which may identify not-grown bacteria potentially contributing to fever, needs improvement in sensitivity, and different reasons for positive PCR in negative blood culture samples need to be assessed before clinical application.

Keywords Bacteremia $\cdot$ Broad-range $\cdot$ Fever $\cdot$ Neutropenia $\cdot$ Polymerase chain reaction

\section{Introduction}

Blood cultures are the gold standard to diagnose bacteremia since about a century. Their potential for further methodological improvement is negligible despite limitations in speed of detection and sensitivity for slow-growing or fastidious organisms or after start of antibacterial therapy [6]. Broad-range polymerase chain reaction (PCR) might offer a promising auxiliary diagnostic tool. It bases on amplifying DNA regions coding for $16 \mathrm{~S}$ or $23 \mathrm{~S}$ rRNA which is highly conserved in all bacteria $[6,7]$. Subsequent sequencing of amplicons allows for identification of bacterial species $[1,6,7]$.

In fever and neutropenia (FN) after chemotherapy, bacteremia is potentially life threatening [8]. The sensitivity and speed limitations of blood cultures are thus especially important. The aim of this two-center pilot study was to assess the applicability and potential problems of broadrange PCR in the daily routine setting by comparing its results with those of routine blood cultures in blood from pediatric cancer patients with FN. 


\section{Materials and methods}

Patients and samples

Cancer patients aged 1 to 17 years presenting with fever (axillary temperature greater than $38.0^{\circ} \mathrm{C}$ for more than $2 \mathrm{~h}$, or greater than $38.5^{\circ} \mathrm{C}$ once) and severe neutropenia (absolute neutrophil count less than $0.5 \times 10^{9} / 1$ ) induced by nonmyeloablative chemotherapy were eligible. Besides oral antiseptic rinsing, antimicrobial prophylaxis was restricted to Pneumocystis jiroveci pneumonia prophylaxis by oral cotrimoxazole or inhaled pentamidine. Multiple FN episodes per patient were allowed. Based on an estimated proportion of $20 \%$ blood culture-positive samples [8], the study aimed at ten blood culture positive episodes to determine the proportion of blood culture-negative but PCR-positive samples with a 95\% confidence interval less than $33 \%$ wide. Patients or their legal guardians gave written informed consent. The respective cantonal ethics committees approved the study.

Before starting empirical intravenous antimicrobial therapy, blood was drawn into a syringe, from which two blood culture bottles and a 5-ml K3E Vacutainer ${ }^{\mathrm{TM}}$ glass tube (Becton Dickinson, Basel, Switzerland) for PCR analysis were filled. Blood cultures were processed in routine automated systems (BACTEC, Becton Dickinson, or BacT/ALERT, bioMérieux, Geneva, Switzerland). The Vacutainer $^{\mathrm{TM}}$ tubes were transported without preprocessing to the laboratory performing broad-range PCR.

Erythrocyte lysis, DNA extraction and real-time broad-range PCR

Lysis of erythrocytes was performed upon receipt of the sample in the laboratory as described [10], and the pellet was stored at $-80^{\circ} \mathrm{C}$ until further processing. The Wizard SV genomic DNA purification system (Promega, Madison, WI) augmented with lysozyme, lysostaphin, and proteinase $\mathrm{K}$, was used for DNA extraction as described [10], the final elution volume being $200 \mu \mathrm{l}$.

Real-time broad-range PCR was performed on $2 \mu \mathrm{l}$ DNA elution to amplify a conserved DNA sequence encoding for the $16 \mathrm{~S}$ rRNA gene using forward primer Bak11W 5'AGTTTGATC(A/C)TGGCTCAG-3', reverse primer Bak2 5'-GGACTAC(C/T/A)AGGGTATCTAAT-3' [5], and probe Tap 5'-CGTATTACCGCGGCTGCTGGCAC-3' [9] with TaqMan ${ }^{\circledR}$ Universal PCR Master Mix and No AmpErase ${ }^{\circledR}$ UNG (Applied Biosystems, Chesire, UK) on an ABI PRISM 7700 Sequence Detector (Applied Biosystems) as described [10]. Each PCR was performed in triplicate. When at least two threshold cycle values were below 38 or one was below 34 [10], results were considered positive if sequencing of the amplicon allowed for bacterial identification and considered indeterminate if sequencing did not allow for identification. Negative controls (blood from healthy volunteer) and positive controls (blood spiked with $10^{6}$ cfu Escherichia coli per PCR) were determined in each run (Fig. 1).

The PCR assay using this specific combination of DNA extraction and primer pair had proven to be the most sensitive of nine assays tested. It detected 18 of 22 bacterial species tested by spiking physiological saline at concentrations of $10^{2} \mathrm{cfu}$ per PCR or below [10]. Applying the probe used together with this primer pair, the sensitivity to detect $E$. coli was identical when spiking blood instead of physiological saline [10].

\section{Sequencing and bacterial identification}

Amplicons were sequenced using primer Bak2 and the BigDye Terminator v1.1 Cycle Sequencing Kit (Applied
Fig. 1 Real-time quantitative broad-range PCR (TaqMan ${ }^{\circledR}$ ) for sample no. 12 . The fluorescence $(\Delta R n)$, measured in each PCR cycle for each sample, is plotted on the $y$-axis against cycle number on the $x$-axis for the sample (\# 12) in triplicate and the positive (pos.; $10^{6} \mathrm{cfu} E$. coli per PCR) and negative controls (neg.; blood from healthy volunteer) each in duplicate. The threshold cycle $\left(C_{\mathrm{T}}\right)$ value corresponds to the cycle number where fluorescence exceeds the $\Delta R n$ threshold set here at $7 \times 10^{-3}$ (horizontal line)

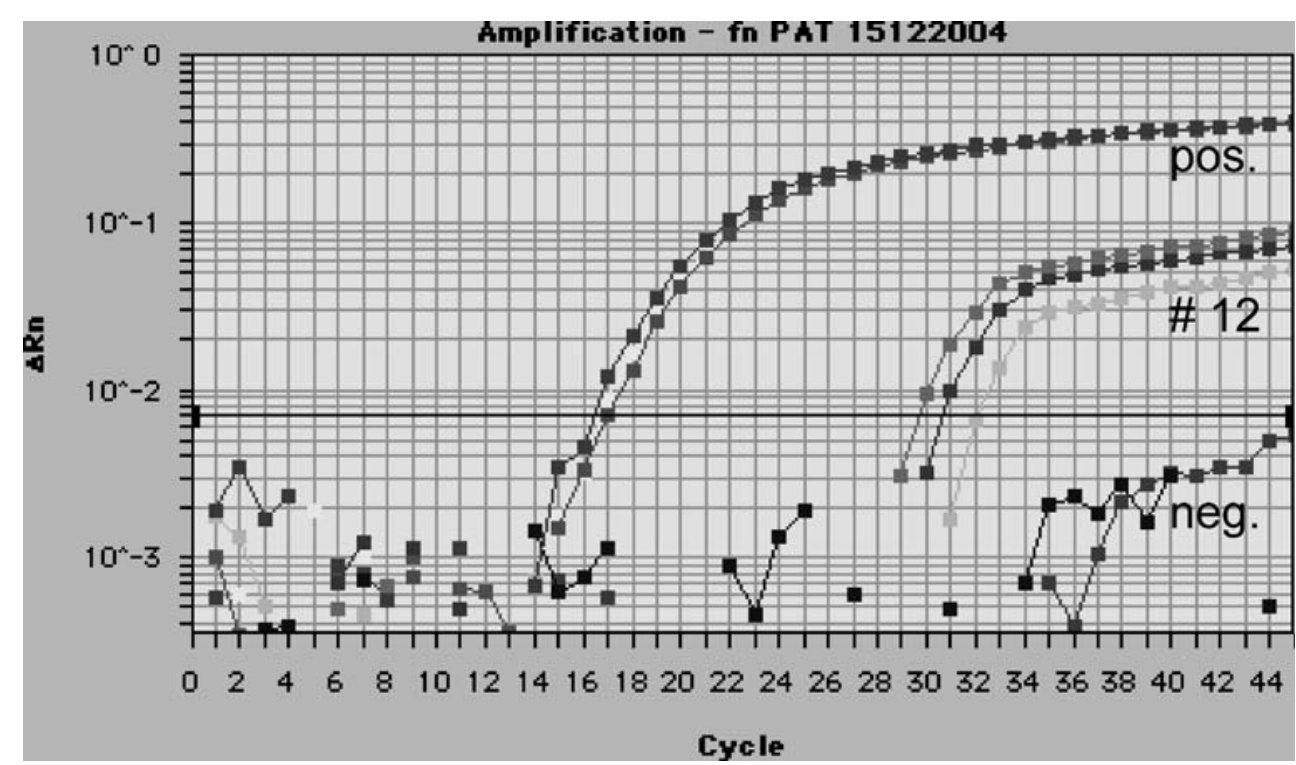


Table 1 Samples with positive blood cultures and/or positive or indeterminate broad-range PCR results ${ }^{\mathrm{a}}$

\begin{tabular}{|c|c|c|c|c|c|}
\hline \multicolumn{2}{|l|}{ Sample } & \multirow{2}{*}{$\begin{array}{l}\text { Blood culture } \\
\text { Result }\end{array}$} & \multicolumn{3}{|c|}{ Broad-range PCR, sequencing, and identification } \\
\hline Number & Age (years) & & Sensitivity ${ }^{\mathrm{b}}$ & Result $^{\mathrm{c}}$ & Identification (score in bits, similarity) \\
\hline 1 & 8.8 & Viridans streptococcus & 10 & Negative & - \\
\hline 6 & 1.4 & Escherichia coli & 1 & Negative & - \\
\hline \multirow[t]{2}{*}{10} & 8.9 & Enterobacter cloacae & 10,000 & Negative & - \\
\hline & & Viridans streptococcus & 10 & & \\
\hline 12 & 2.7 & CoNS & 1 & Positive & Staphylococcus epidermidis $(444,96 \%)$ \\
\hline \multirow[t]{2}{*}{15} & 13.5 & CoNS & 1 & Negative & - \\
\hline & & Bacillus fragilis & 100 & & \\
\hline 16 & 13.5 & B. fragilis & 100 & Indeterminate & - \\
\hline 26 & 2.8 & CoNS & 1 & Indeterminate & - \\
\hline 27 & 15.6 & $\mathrm{CoNS}^{\mathrm{c}}$ & 1 & Negative & - \\
\hline 38 & 13.2 & Klebsiella pneumoniae ${ }^{\mathrm{d}}$ & 10,000 & Positive & Klebsiella pneumoniae $(488,92 \%)$ \\
\hline \multirow[t]{2}{*}{41} & 3.0 & K. pneumoniae & 10,000 & Positive & Stenotrophomonas maltophilia $(438,91 \%)$ \\
\hline & & Pseudomonas aeruginosa & 100 & & \\
\hline 2 & 1.7 & Negative & NA & Positive & Pseudomonas aerigompsa $(82,88 \%)$ \\
\hline 3 & 4.5 & Negative & NA & Positive & S. maltophilia $(174,84 \%)$ \\
\hline \multirow[t]{3}{*}{9} & 6.7 & Negative & NA & Positive & Pseudomonas spp. (466, 92\%) \\
\hline & & & & & S. maltophilia $(462,92 \%)$ \\
\hline & & & & & Acinetobacter calcinoaceticus $(462,92 \%)$ \\
\hline 18 & 2.8 & Negative & NA & Indeterminate & - \\
\hline \multirow[t]{2}{*}{19} & 9.5 & Negative & NA & Positive & S. maltophilia $(527,94 \%)$ \\
\hline & & & & & A. calcinoaceticus $(527,94 \%)$ \\
\hline 20 & 13.0 & Negative & NA & Indeterminate & - \\
\hline 21 & 13.8 & Negative & NA & Positive & S. maltophilia $(492,93 \%)$ \\
\hline 23 & 4.1 & Negative & NA & Positive & S. maltophilia $(206,85 \%)$ \\
\hline 25 & 4.7 & Negative & NA & Positive & S. maltophilia $(357,89 \%)$ \\
\hline 30 & 3.1 & Negative & NA & Positive & S. maltophilia $(258,87 \%)$ \\
\hline 31 & 2.9 & Negative & NA & Positive & S. maltophilia $(76,87 \%)$ \\
\hline 40 & 3.8 & Negative & NA & Positive & S. maltophilia $(145,84 \%)$ \\
\hline 43 & 5.0 & Negative & NA & Indeterminate & - \\
\hline 44 & 4.9 & Negative & NA & Indeterminate & - \\
\hline
\end{tabular}

${ }^{a}$ In 21 episodes of fever and neutropenia, results of both methods were negative (median age at sampling, 11.2 years)

${ }^{\mathrm{b}}$ Analytical sensitivity (cfu per PCR) of this combination of DNA extraction and PCR for species detected in water [10]

${ }^{\mathrm{c}}$ Positive, if at least two threshold cycle $\left(C_{\mathrm{T}}\right)$ values below 38 or at least one $C_{\mathrm{T}}$ value below 34 and bacterial identification possible by sequencing the amplicon; indeterminate if at least two threshold cycle $\left(C_{\mathrm{T}}\right)$ values below 38 or at least one $C_{\mathrm{T}}$ value below 34 but no bacterial identification by sequencing; negative in the remaining samples

d Two different strains isolated

CoNS indicates coagulase-negative staphylococcus; $N A$ not applicable, as blood cultures were negative

Biosystems). The samples were analyzed on an ABI PRISM 310 Genetic Analyzer (PerkinElmer, Boston, MA) as described [10]. Bacterial identification was done in a two-step procedure as described [1, 10]. First, the FASTA algorithm of the GCG Wisconsin software package (Accelrys) with visual inspection of the electropherogram, and sequence correction where necessary was performed. It was followed by a second search using the basic local alignment search tool (http://www.ncbi.nlm.nih.gov/blast). Criteria for identification to the genus or species level were used as described [1]. For each sequence, only the highestscoring and, if applicable, the next highest-scoring species were recorded.
Statistics

StatXact 6.3.0 (Cytel, Cambridge, MA) was used for Zelen's exact test of homogeneity of odds ratios, and for the exact Mann-Whitney test, where applicable.

\section{Results}

Blood cultures detected bacteremia in $10(22 \%)$ of $45 \mathrm{FN}$ episodes in 31 patients with FN recruited consecutively in two centers during a 6-month period in 2003. Patient's median age at first FN was 8.8 years (range, 1.4-16.5). 
Diagnoses included acute lymphoblastic leukemia in 14, acute myeloid leukemia in two, lymphoma in five, and solid tumors in ten patients.

Broad-range PCR, performed after optimization of the method [10] in 2005, was positive in three and indeterminate in two of ten blood culture-positive samples and positive in 10 and indeterminate in 4 of 35 blood culturenegative samples (Table 1). There were no significant associations of these ratios with center, diagnostic group, fever rule, and peripheral versus central venous access (Zelen's test; data not shown). Among patients with blood culture-negative samples, a positive versus negative PCR result was significantly associated with younger age (median, 4.3 versus 11.2 years; Mann-Whitney statistic 394, $p=0.013$ ).

In the three samples with both positive blood culture and PCR results, bacterial identification confirmed the species detected in blood cultures in two samples (Staphylococcus epidermidis and Klebsiella pneumoniae), whereas in the third sample, growing K. pneumoniae and Pseudomonas aeruginosa in blood cultures, Stenotrophomonas maltophilia was identified.

In nine of the ten samples with negative blood culture but positive PCR result, S. maltophilia with or without other species (Acinetobacter calcoaceticus and Pseudomonas spp.) was identified. The sequences of these nine samples all differed from each other (data not shown). Pseudomonas aerigompsa was identified in one sample.

\section{Discussion}

In contrast to the results of the study that had established the specific assay of broad-range PCR used [10], the sensitivity of real-time broad-spectrum PCR was not high in this pilot study. Two main reasons might explain this. First, the analytical sensitivity of the applied combination of DNA extraction and PCR protocol ranges, depending on the bacterial species, between $1 \mathrm{cfu}$ and 10,000 per PCR in physiological saline [10]. However, there was no evident association between sensitivity and result of broad-range PCR (Table 1). In contrast, the blood culture method may, at least theoretically, detect bacteremia with only $1 \mathrm{cfu}$ per blood culture bottle for each species growing in this system. Second, bacterial blood loads are expected to be lower after nonmyeloablative than after myeloablative chemotherapy, where sensitivity of broad-range PCR reported is higher [3, 6]. In the three PCR- and blood culture-positive samples, species identification was correct in two samples, whereas the apparent discrepancy in the third sample likely resulted from the high agreement of $16 \mathrm{~S}$ sequences of $P$. aeruginosa grown in culture and $S$. maltophilia (formerly classified as Pseudomonas maltophilia) identified by PCR.
Broad-range PCR was positive in $29 \%$ of the samples with negative blood culture, which is comparable to published results (up to $42 \%$ ) $[4,6]$. As broad-range PCR aims at detection of additional cases of bacteremia compared to blood cultures as the nonperfect gold standard, this proportion cannot be converted into a specificity. In nine of ten blood culture-negative but PCR-positive samples, S. maltophilia was identified, twice together with A. calcoaceticus and once with Pseudomonas spp, whereas $P$. aerigompsa was identified once. These ubiquitous gramnegative aerobe rods that can cause nosocomial infections are known to grow well in routine blood culture systems. Thus, it remains unclear to which entity the positive PCR result in samples with negative blood culture correspond to. First, they might represent contamination, an inherent drawback in PCR-based methods $[2,6]$. The fact that the sequences of the nine samples with $S$. maltophilia identified all differed from each other strongly argues against laboratory contamination. Second, they might correspond to clinically not relevant background bacterial DNA, i.e., residues of dead or degraded bacteria, reported in the blood of healthy subjects $[4,6]$. Background DNA of $S$. maltophilia and Pseudomonas spp. has been described [4]. Third, they might represent the clinically relevant additional detection of bacterial infection, e.g., of bacterial compounds spilled over from a localized infection into the blood stream, not detected by blood culture. Another reason for the failure to detect $S$. maltophilia in blood cultures might have been the routine prophylactic application of oral trimethoprim-sulfamethoxazole to prevent $P$. jiroveci pneumonia, as this antimicrobial shows activity against most strains of $S$. maltophilia. In a different setting, namely, in patients receiving intravenous antibacterial therapy, the detection of Pseudomonas spp. and Acinetobacter spp. by PCR in samples with negative blood cultures has been described [3].

The statistically significant younger patient age in blood culture-negative samples with positive versus negative PCR might result from greater bacteremia in younger children, or it might reflect younger children showing less containment of local bacterial infections than older children. Thus, the identification of bacteria in blood samples showing no growth in cultures by broad-range PCR could help identify neutropenic patients developing fever because of a bacterial infection. Such information could assist in the devise of empiric antimicrobial treatment in FN.

In conclusion, this pilot study demonstrates that realtime broad-range PCR may be worth further studying in FN because of its potential advantages over blood cultures including faster detection and identification within one to two working days [10], quantitative results allowing longitudinal assessment and better sensitivity for slowgrowing or fastidious organisms, or after antimicrobial 
therapy [6]. Large prospective studies are needed to optimize its performance and then to assess its final clinical value. Less than optimal sensitivity, the inherent potential for contamination, and the difficulty to distinguish background DNA from real bacteremia seem to be the main drawbacks at this moment, with blood culture remaining as the reference test. Possible optimizations are preincubation in blood culture media to enhance sensitivity, nested PCR to reduce the risk of contamination and to allow for sequencing in infection with multiple germs [3], and the simultaneous detection of DNA coding for 16S rRNA and the corresponding rRNA itself as a measure of viability [6].

Acknowledgments We acknowledge Maja Beck Popovic, MD, Lausanne, Annette Ridolfi Lüthy, MD, Bern, and Pierre Wacker, MD, Geneva, members of the SPOG 2003 FN Pilot Eubacterial PCR Study Group Committee, for contributions in designing the study protocol, Andrea Wasem, RN, and Nadine Beusch, RN, for assistance in data collection, and Sibylle Gunziger and Gudrun Blessing for technical assistance.

\section{References}

1. Bosshard PP, Kronenberg A, Zbinden R, Ruef C, Bottger EC, Altwegg M (2003) Etiologic diagnosis of infective endocarditis by broad-range polymerase chain reaction: a 3-year experience. Clin Infect Dis 37:167-172

2. Corless CE, Guiver M, Borrow R, Edwards-Jones V, Kaczmarski EB, Fox AJ (2000) Contamination and sensitivity issues with a real-time universal 16S rRNA PCR. J Clin Microbiol 30:17471752

3. Ley BE, Linton CJ, Bennett DM, Jalal H, Foot AB, Millar MR (1998) Detection of bacteraemia in patients with fever and neutropenia using $16 \mathrm{~S}$ rRNA gene amplification by polymerase chain reaction. Eur J Clin Microbiol Infect Dis 17:247-253

4. Nikkari S, McLaughlin IJ, Bi W, Dodge DE, Relman DA (2001). Does blood of healthy subjects contain bacterial ribosomal DNA? J Clin Microbiol 39:1956-1959

5. Goldenberger D, Kunzli A, Vogt P, Zbinden R, Altwegg M (1997) Molecular diagnosis of bacterial endocarditis by broad-range PCR amplification and direct sequencing. J Clin Microbiol 35:27332739

6. Peters RPH, van Agtmael MA, Danner SA, Savelkoul PHM, Vandenbroucke-Grauls CMJE (2004) New developments in the diagnosis of bloodstream infections. Lancet Infect Dis 4:751760

7. Song JH, Cho H, Park MY, Na DS, Moon HB, Pai CH (1993) Detection of Salmonella typhi in the blood of patients with typhoid fever by polymerase chain reaction. J Clin Microbiol 31:1439-1443

8. Walsh TJ, Roilides E, Groll AH, Gonzalez C, Pizzo PA (2006) Infectious complications in pediatric cancer patients. In: Pizzo PA, Poplack DG (eds) Principles and practice of pediatric oncology, 5th edn. Lippincott Williams \& Wilkins, Philadelphia, pp 12691329

9. Warwick S, Wilks M, Hennessy E, Powell-Tuck J, Small M, Sharp J, Millar MR (2004) Use of quantitative 16S ribosomal DNA detection for diagnosis of central vascular catheter-associated bacterial infection. J Clin Microbiol 42:1402-1408

10. Zucol F, Ammann RA, Berger C, Aebi C, Niggli FK, Nadal D (2006) Real-time quantitative broad-range PCR assay for detection of the 16S rRNA gene followed by sequencing for species identification. J Clin Microbiol 44:2750-2759 